\title{
A three-step approach to patients with suspected acute diverticulitis in the emergency department: An interdisciplinary algorithm proposal
}

\author{
Ciro Paolillo, ${ }^{1}$ Massimo Sartelli, ${ }^{2}$ Vittorio Miele,${ }^{3}$ Luca Ansaloni, ${ }^{4}$ Massimo Valentino, ${ }^{5}$ \\ Cecilia Merli, ${ }^{6}$ Federico Coccolini, ${ }^{4}$ Francesco Toso, ${ }^{7}$ Carmelo Madia, ${ }^{8}$ Fausto Catena ${ }^{9}$
}

${ }^{1}$ Emergency Department, Spedali Civili, Brescia; ${ }^{2}$ Department of Surgery, Macerata Hospital, Macerata; ${ }^{3}$ Department of Radiology, Careggi University Hospital, Firenze; ${ }^{4}$ General, Emergency and Trauma Surgery Department, Bufalini Hospital, Cesena; ${ }^{5}$ Department of Radiology, Tolmezzo Hospital, Tolmezzo; ${ }^{6}$ Emergency Department, Bufalini Hospital, Cesena; ${ }^{7}$ Department of Radiology, Teaching Hospital, Udine; ${ }^{8}$ Emergency Department, Treviso Hospital, Treviso; ${ }^{9}$ Department of Emergency Surgery, Parma Maggiore Hospital, Parma

\begin{abstract}
Patients with acute abdominal pain due to acute colonic diverticulitis are a frequent cause of hospital admission. Diverticuar disease increases with age. In Western populations, diverticular disease is diagnosed in $50 \%$ individuals over 70 years and $80 \%$ over 85 years of age. The identification of patients with acute diverticulitis is a considerable challenge, since numerous other acute abdominal conditions mimic its clinical characteristics. Another problem is the atypical presentation in older patients. A high index of suspicion in the setting of a compatible history and physical examination serves as the cornerstone of early diagnosis. The purpose of this review is to underline the importance of an intimately integrate multidisciplinary approach between emergency physicians, radiologists and surgeons.
\end{abstract}

\section{Introduction}

Diverticular disease in the emergency department

Acute abdominal pain is the most common cause for visits to

Correspondence: Ciro Paolillo, Emergency Department, Spedali Civili, Piazzale Spedali Civili 1, Brescia, Italy.

Tel.: +39.0303995818.

E-mail: ciropaolillo@gmail.com

Key words: Acute colonic diverticulitis; Clinical diagnosis; Abdominal pain; Diagnostic accuracy; Imaging modality; Abdominal ultrasound; Computed tomography.

Contributions: the authors contributed equally.

Conflict of interest: the authors declare no potential conflict of interest.

Funding: none.

Received for publication: 12 February 2018.

Revision received: 12 June 2018.

Accepted for publication: 13 June 2018.

This work is licensed under a Creative Commons Attribution 4.0 License (by-nc 4.0).

(C) Copyright C. Paolillo et al. 2018

Licensee PAGEPress, Italy

Emergency Care Journal 2018; 14:7339

doi:10.4081/ecj.2018.7339 the emergency department (ED), accounting for an estimated 5$10 \%$ of all admissions. ${ }^{1}$ The underlying causes of abdominal pain range from life-threatening conditions that require fairly immediate decision and urgent surgical intervention to self-limiting conditions from common to unusual diseases. Abdominal pain is a common occurrence in elderly patients. With the increasing mean age of the population, the prevalence of age-correlated diseases has concomitantly increased. ${ }^{2}$ A typical example is acute diverticulitis (AD). In Western populations, aging and lifestyle changes place the elderly at high risk for diverticulosis. Diverticula are a sac-like outpouchings of the colon that occur through weakened areas of the muscularis of the colon wall. The pathophysiology is not well understood but diets low in fiber are epidemiologically related to the underlying process of outpouching of colonic mucosa. ${ }^{3}$ Estimated at $5 \%$ of general population, diverticular disease is diagnosed in $50 \%$ individuals over 70 years and $80 \%$ over 85 years of age. ${ }^{2}$ A population-based cohort study on the Danish population from 2000 to 2012 evidenced that overall admission rates for complicated diverticulitis increased significatly. ${ }^{4}$ The most frequent complications from diverticula include bleeding and diverticulitis. Bleeding occurs in $15 \%$ of patients with diverticulosis. It is the most common cause of lower gastrointestinal bleeding in the elderly. The bleeding is usually mild, but occasionally it is massive. Several studies have reported that approximately $30 \%$ of patients with diverticular disease develop diverticulitis or its complications. ${ }^{1}$ Diverticulitis is caused by obstruction or abrasion by a fecalith, leading to inflammation, and eventually infection from the proliferation of colonic bacteria and build-up of bowel secretions within the diverticula. According to an earlier Swedish study, about 47 patients per 100,000 population/year are admitted in the $\mathrm{ED}$ for $\mathrm{AD} .^{5}$ It occurs most frequently in the descending and sigmoid regions of the colon but can occur throughout the colon.

\section{The clinical problem}

The most common symptom of diverticulitis is abdominal pain. The pain usually evolves over 1 to 2 days from dull, diffuse abdominal discomfort to more intense, localized left lower quadrant pain. Classic signs of left lower quadrant abdominal pain associated with cramping, fever, nausea with occasional vomiting, mild abdominal distention, decreased appetite and leukocytosis are usually present. Many may report a known history of diverticular disease. Diarrhoea and constipation have both been reported as alterations in bowel habits. Diverticulitis might be complicated by the formation of an abscess or fistula, bowel obstruction, free perforation, or the development of sepsis.

The identification of patients with AD is a considerable chal- 
lenge, since numerous other acute abdominal conditions can mimic its clinical characteristics. The left lower quadrant is more frequently involved in $\mathrm{AD}$, such as urolithiasis, acute appendicitis, stercoral perforation, ischemic or infectious colitis, aortic abdominal aneurysm, spontaneous retroperitoneal hemorrhage or gynecological disease (Table 1). ${ }^{6}$ In 2010, Lameris et al. monitored 126 patients with suspected diverticulitis and showed different final diagnoses in $37 \%$ of cases (aspecific abdominal pain and acute appendicitis at first). ${ }^{7}$ In contrast to appendicitis, diagnostic value of specific elements has not been extensively studied for AD. A comprehensive study by the group of Andeweg characterized patients with abdominal pain at high risk of AD based on the following criteria: age greater than 50 years, one or more similar previous episodes, localisation of symptoms in the lower left abdomen, aggravation of pain on movement, absence of vomiting, localisation of tenderness in the lower left abdomen, and CRP of $50 \mathrm{mg} / \mathrm{L}$ or higher. ${ }^{6}$ The discriminatory power of the different variables alone was very low but improved upon combining the independent risk factors. The sensitivity of clinical evaluation was not satisfactory, with accuracy in only $43 \%$ of patients from the Andeweg cohort. Other comparable studies have demonstrated similar low sensitivity. ${ }^{5}$

In elderly patients many factors make diagnosis of $\mathrm{AD}$ even more difficult. Perception of pain is often suppressed due to decreased immune responses and changes in the neurological system. Additionally, muscular defense and rebound signs in abdominal examination may not be evident despite the presence of serious intra-abdominal diseases because of a thinner abdominal wall, degeneration of the inflammatory process, and abolition of peripheral nerve functions. Thirty percent of elderly with AD don't have abdominal tenderness on exam. Immunosenescence is associated with aging. Older adults experience a reduced febrile response caused by altered thermoregulation and decrease in mean body temperature. Laboratory studies have significant limitations, particularly in olders. The group of Laurell (2006) reported that laboratory parameters cannot be effectively used to distinguish patients affected by $\mathrm{AD}$ from those with aspecific abdominal pain. ${ }^{8}$ Overall, 24 patients $(16 \%)$ with a final diagnosis of diverticulitis showed a CRP level below the upper limit of the 95\% confidence interval relative to CRP of patients with a final diagnosis of aspecific abdominal pain. Same limitations were evident for leukocyte count. A recent study by the group of Lameris showed that the strongest clinical features used for diagnosis of $\mathrm{AD}$ (tenderness in the left lower quadrant, absence of vomiting and CRP level $>50$ $\mathrm{mg} / \mathrm{L}$ ) were only present in about a quarter of patients. ${ }^{7}$ Almost half of older patients suffering AD have a normal white blood cell count.

Diagnosis of AD involves a strategy encompassing age, history, clinical, differential diagnosis and laboratory tests but often it's not enough. It's necessary increase in accuracy after clinical evaluation following an imaging strategy.

\section{An imaging strategy}

In the setting of suspected $\mathrm{AD}$, the clinical questions are essentially the following: i) to exclude other potential diseases that can mimic diverticulitis; ii) to confirm the diagnosis of diverticulitis and iii) to stage the entity of the disease.

Abdominal radiography is of limited value, and cannot be utilised to achieve either of the above objectives. Furthermore, plain radiography may be applied in case of suspicion of diverticulitis complicated by occlusion or perforation but it's $100 \%$ sensitive only for a large pneumoperitoneum. ${ }^{9}$ Ultrasound (US) is a useful imaging modality for evaluating the abdomen, being noninvasive, portable, widely available, and relatively inexpensive. US, even in the hands of an emergency physician, is highly accurate and sensitive in identifying the most frequent diseases that can overlap with AD (abdominal aortic aneurysms, obstructive hydronephrosis, intestinal occlusion). US findings in patients with AD include a thickened loop of bowel with a target-like appearance. Small air bubbles can be visualised as a sign for microperforation. Sonography is also useful for assessing large abscesses. In emergency setting the most common US technique used to examine patients with suspected $\mathrm{AD}$ is a transabdominal compression, first introduced in 1990. The clinician performs an abdominal US to assess for diverticulitis using a low-frequency curvilinear transducer by gently squeezing through loops of the bowel in the area of localised abdominal pain. The presence of echogenic non-compressible fat surrounding one or more diverticula is suggestive for the presence of inflammation. ${ }^{10-12}$

The sensitivity of US in diagnosing AD has been reported to be as high as $84-100 \% .{ }^{13}$ But two complete and comprehensive literature researches conducted by the groups of Liljegren in $2006^{14}$ and Lameris in $2008^{13}$ to compare the diagnostic accuracy of graded compression of US $v s$ CT in diagnosis AD revealed a very limited number of high-quality studies. A complete search of the Medline, Embase and Cinahl databases resulted in a collection of 1689 studies, among which only 12 were relevant. Numerous bias

Table 1. Differential diagnosis of left lower quadrant pain.

$\begin{array}{lcc}\text { Vascular } & \text { Gastrointestinal } & \text { Genitourinary } \\ \text { Abdominal aneurysm } & \text { Acute left colon diverticulitis } & \text { Ureterolithiasis } \\ \text { Iliacal aneurysm } & \text { Colorectal malignancy } & \text { Urinary infection } \\ \text { Urinary malignancy } & \text { Intestinal ischemia } & \text { Other } \\ \text { Gynecologic } & \text { Incarcerated hernia } & \text { Constipation } \\ \text { Ectopic pregnancy } & \text { Diverticulosis } & \text { Petroperitoneal hemorrhage } \\ \text { Malignancy } & \text { Acute appendicitis } & \text { Aspecific abdominal pain } \\ \text { Ovarian torsion } & \text { Intestinal obstruction } \\ \text { Ruptured corpus luteum } & \text { Sigmoid volvulus } \\ \text { Ruptured ovarian cyst } & \text { Mesenteric lymphadenitis } \\ \end{array}$


factors (for instance, representative heterogeneity of the population, non-specific selection criteria, inaccurate description of the reference standard) affected the results in most of the studies. Only two investigations performed a head-to-head comparative accuracy assessment of US and CT. In both cases, the two imaging modality displayed similar accuracy in evaluation of patients suspected with diverticulitis. However, both studies were single-centre trials, including only 63 patients recruited by Soliman ${ }^{15}$ and only 64 by Pradel. ${ }^{16}$ In both cases, the images were had been interpreted by radiologists. In 1997 , Zielke et al. ${ }^{17}$ reported another similar results. The group calculated the sensitivity and specificity of US performed by surgeons on a small group of 74 patients with suspected $\mathrm{AD}$. Their results were encouraging, disclosing a sensitivity of $84 \%$ and specificity of $93 \%$, but the same numerous bias factors remained to be taken into account. It must be noted that these studies were not designed for the emergency room arena and are potentially misleading, providing a false impression of hightest accuracy. If utilized by providers with appropriate training, US could possibly represent an ideal first-line imaging test for uncomplicated diverticulitis. Accordingly, a step-up approach has been proposed that involves validation with $\mathrm{CT}$ after an inconclusive or negative US results. ${ }^{18}$ The majority of Experts agree that the accuracy of US is limited in obese patients when inflammation is minimal or air is present in the colon, and correlated with the examiner's expertise. ${ }^{19-37}$ Multicentric study will certainly be needed of focus on the role of US. The American College of Radiology recommends abdominal and pelvic CT with IV contrast (rating 9) in patients with suspected AD. ${ }^{19}$ The TC responds accurately to all 3 questions we have seen above: it is useful to evaluate the severity and extent of disease and to identify complications, it also may diagnose other causes of left lower-quadrant pain that can mimic diverticulitis. ${ }^{20} \mathrm{CT}$ has reported sensitivity and specificity as high as 100 percent in diagnosing acute sigmoid diverticulitis. ${ }^{21}$ According with old studies published by BC Morson from St Mark's Hospital in London in $1963^{22}$ the mesenteric fat tissue around the sigmoid colon plays a central role in diagnosing and managing an $\mathrm{AD}$. If the mesenteric fat becomes inflammed can produce a phlegmon or an abscess. Studying the fat around the sigmoid colon helps to determine the severity of disease, from here the treatment planning. ${ }^{23}$ In the real world the choice of imaging strategies are driven by the clinical and by patient's characteristics. In obese patient, in whom US is more likely to be non diagnostic, in older patients with a new acute left lower abdominal pain and in severely ill patients the $\mathrm{CT}$ is more able to detecting alternative diagnoses and to assist in planning of a radiological or surgical intervention.

\section{The management}

In the last years the management of $\mathrm{AD}$ is profoundly changed. Until a few years ago hospitalization, bowel rest, intravenous fluids and antibiotic therapy were universally accepted as the first choice treatment for mild uncomplicated diverticulitis. This practice was based on the belief that diverticulitis was due to obstruction of a diverticulum leading to mucosal abrasions, microperforation and bacterial translocation. However, this concept has been changed with newer hypotheses highlighting that $\mathrm{AD}$ may be an inflammatory rather than an infectious condition. Indeed, prospective randomized, case-control and retrospective cohort study shown no benefit for the use of antibiotics in the treatment of uncomplicated $\mathrm{AD}$, suggesting that its use should be reserved for the treatment of complicated disease. ${ }^{23-25}$ An increasingly conservative approach changed also the surgery. The old simple rule of 2 attacks, then operate is gone, the patients with pericolic air or small fluid collection should be managed by antibiotic therapy, abscesses having a diameter of more than $4 \mathrm{~cm}$ are best treated by percutaneous drainage. ${ }^{26}$ Surgical resection is confined to patients with severe sepsis or septic shock, or if non-invasive treatments fail. The cornerstone is to create a puzzle that takes in account the clinical conditions and the extension of the disease. ${ }^{26}$ More extensive is the infection, more aggressive is the treatment. Fortunately, the majority of $\mathrm{AD}$ episodes are uncomplicated, with about only $15 \%$ presenting complications (abscess, perforation, obstruction, fistula); even if a recurrence of AD has been reported in $15-30 \%$ of patients, generally the first episode is the most severe. ${ }^{27-29}$ The development of CT has radically changed the treatment approach for patients with acute diverticulitis. CT can be effectively employed not only to diagnose colonic acute diverticulitis but also verify the severity and extent of disease. Staging of acute diverticulitis is an essential tool that could aid in clinical decision making, such as determining the need for percutaneous drainage or surgical intervention, assessing the length of hospitalization and antibiotic administration or the opportunity for early return home directly from the ED. The original classification proposed by Hinchey ${ }^{38}$ in 1978 was used as an intraoperative system, and did not meet these needs. The modified Hinchey classification proposed in $1999^{39}$ was essentially a surgical assessment that described the stages of perforated diverticular disease. Both classifications have some bias, they don't take clinical symptoms into account, and are built for advanced-stage disease. In 2015, Sartelli et al. ${ }^{26}$ proposed a more complete classification taking in account the severity of the disease and the clinical condition (Table 2). Fortunately, patients with uncomplicated diverticulitis are more frequently admitted to the ED. Usually the patient complains of moderate pain and is able to tolerate oral intake, with good response to first treatment measures in the ED. A corroborative CT is performed in these cases, which highlights that the infection only involves the colon and does not extend to the peritoneum, leading to safe discharge of patients directly from the ED. Furthermore, evidence of involvement of $<5 \mathrm{~cm}$ of the colon is associated with a low probability of recurrence. ${ }^{40}$ The severity of diverticulitis on CT is statistically predictive of the risk of medical treatment failure during the acute phase and chance of poor secondary outcome after successful medical treatment of the first episode. Staging is also a guide for antibiotic therapy. The AVOD study was the first prospective random-

Table 2. A proposal for a computed-tomography guided classification of acute diverticulitis. Adapted from Sartelli $e t$ al. ${ }^{26}$

\begin{tabular}{ll}
\hline Stage 0 & Diverticula, thickening of the colonic wall or increased density of the pericolic fat \\
Stage la & Pericolic air bubbles or little pericolic fluid without abscess (within $5 \mathrm{~cm}$ from inflamed bowel segment) \\
\hline Grade Ib & Abscess $\leq 4 \mathrm{~cm}$ \\
Grade IIa & Abscess $>4 \mathrm{~cm}$ \\
\hline Grade IIb & Distant air $(>5 \mathrm{~cm}$ from inflamed bowel segment) \\
\hline
\end{tabular}


ized controlled trial to show no benefit of antibiotic treatment in patients with uncomplicated diverticulitis. ${ }^{41}$ Small abscesses may be solely treated with antibiotics while large abscesses need to be interventionally drained in addition to antibiotic treatment.

\section{The three-step approach}

Several guidelines and reviews on diverticular disease have been published in lasts years with discrepancies regarding important issues, such as the role of clinical examinations, the imaging approach, the antibiotic prescriptions for uncomplicated diverticulitis, treatment of abscesses, and indications for colon resection in case of peritonitis. ${ }^{20,30-34}$ The diversified approach is attributed to the lack of high-quality evidence. ${ }^{30}$ Another limitation of these guidelines is that they were originally developed by experts in various fields, including gastroenterologists, ${ }^{31,32}$ colorectal surgeons $^{33,34}$ and emergency surgeons. ${ }^{23}$ In the clinic, the course of suspected $\mathrm{AD}$ can involves emergency physicians at first, followed if necessary by radiologists and surgeons. Aim of this review authored by a small group of emergency physicians, radiologists and surgeons with expertise in acute abdominal pain is focused on a multimodal and collaborative approach for treatment of patients suspected of AD. This approach involves three steps, each of them is essential for the success of the course.

\section{Step one: the suspicion}

i) Age greater than 50 years, one or more similar previous episodes, localization of symptoms in the lower left abdomen, aggravation of pain on movement, absence of vomiting, tenderness in the lower left abdomen, and CRP of $50 \mathrm{mg} / \mathrm{L}$ or higher characterize patients with high suspect of AD; ii) It's reasonable a prudent approach and an high suspect of disease in older patients with lower abdominal pain. Difficulty in obtaining history, lack of consistent physiologic responses (fever, hypotension, pain, tachycardia and leukocytosis) make diagnosis of AD a very challenge; iii) Since clinical assessment remains ambiguous in the majority of cases, an imaging strategy is recommended.

\section{Step two: the confirmation}

i) US may aid in excluding the most frequent diseases that mimic of $\mathrm{AD}$, (abdominal aortic aneurysm or hydronephrosis, for example); ii) US appears also to present a logical and safe technique of choice in younger skinny patients or if there's the suspect of a non-complicated recurrent colonic diverticulitis; iii) CT is the imaging technique of choice for evaluation of patients with suspected AD. CT is also helpful in identifying alternative diagnoses with similar presentations.

\section{Step three: the staging}

i) Assessing the site and the severity of the disease is the key; ii) Staging of diverticulitis on $\mathrm{CT}$ is the guide for the treatment planning according to its severity; iii) Effective communication and condivision of strategies among emergency physicians, radiologists and surgeons must be necessary for a positive impact on the quality of care provided to patients with suspect of $\mathrm{AD}$.

\section{Conclusions}

Over the last 10 years, the number of patients suffering of $A D$ is increasing in all over the world. ${ }^{42}$ It is now classified as the third most common gastrointestinal discharge diagnosis. ${ }^{43}$ Most of the time signs and symptoms associated with $\mathrm{AD}$ are aspecific and commons to other intra-abdominal pathologic conditions. In recent years, integrated imaging has revolutionized the classical approach to acute abdominal pain and close cooperation is essential among emergency physicians, radiologists and surgeons for improving treatment outcomes. A high index of suspicion in the setting of a compatible history and physical examination is the cornerstone of early diagnosis. Inclusion of an appropriate diagnostic modality is crucial to validate initial suspicion of the disease, to exclude other potential diseases that mimic diverticulitis, and to evaluate the severity and extent of disease. Only a collaborative strategy that takes into account the extension of the disease, the clinical condition and the compliance of the patients permits to plan a tailored treatment.

\section{References}

1. Chang CC, Sun-Sang W. Acute abdominal pain in the elderly. Int J Geront 2007;1:77-82.

2. Martinez JP, Mattu A. Abdominal pain in the elderly. Emerg Clin N Am 2006;24:371-88.

3. Hobson KG, Roberts PL. Etiology and pathophysiology of diverticular disease. Clin Colon Rectal Surg 2004;17:147-53.

4. Hupfeld L, Pommergaard HC, Burcharth J, Rosenberg J. Emergency admissions for complicated colonic diverticulitis are increasing: a nationwide register-based cohort study. Int J Colorectal Dis 2018;33:879-86.

5. Lehtimaki TT. Detecting acute mesenteric ischemia in CT of the acute abdomen is dependent on clinical suspicion: Review of 95 consecutive patients. Eur J Radiol 2015;84:2444-53.

6. Andeweg CS, Knobben L, Hendriks JCM, et al. How to diagnose acute left-sided colonic diverticuitis. Proposal for a clinical scoring system. Ann Surg 2011;253:940-6.

7. Lameris W, van Randen A, van Gulik TM, et al. A clinical decision rule to establish the diagnosis of acute diverticulitis at the Emergency Department. Dis Colon Rectum 2010;53:896-904.

8. Laurell H, Hansson LE, Gunnarsson U. Acute diverticulitis clinical presentation and differential diagnostics. Colorectal Dis 2007;9:496-502.

9. Kumar A. The etiology of pneumoperitoneum in the 21 st century. J Trauma Acute Care Surg 2012;73:542-8.

10. Schwerk WB, Schwarz S, Rothmund M. Sonography in acute colonic diverticulitis. A prospective study. Dis Colon Rectum 1992;35:1077-84.

11. Wilson SR, Toi A. The value of sonography in the diagnosis of acute diverticulitis of the colon. AJR Am J Roentgenol 1990;154:1199-202.

12. Mazzei MA, Cioffi Squitieri N, Guerrini S, et al. Sigmoid diverticulitis: US findings. Crit Ultrasound J 2013;5:S5.

13. Lameris W. Graded compression ultrasonography and computed tomography in acute colonic diverticulitis: Meta-analysis of test accuracy. Eur Radiol 2008;18:2498-511.

14. Liljegren G, Chabok A, Wickbom M, et al. Acute colonic diverticulitis: a systematic review of diagnostic accuracy. Colorectal Dis 2007;9:480-8.

15. Soliman F. Primary diagnostics of acute diverticulitis of the sigmoid. Ultraschall Med 2004;25:342-7.

16. Pradel JA. Acute colonic diverticulitis: prospective compartive evaluation with US and CT. Radiology 1997;205:503-12.

17. Zielke A, Hasse C, Nies C, et al. Prospective evaluation of ultrasonography in acute colonic diverticulitis. Br J Surg 
1997;84:385-8.

18. Lameris W, van Randen A, van Es HW, et al. Imaging strategies for detection of urgent conditions in patients with acute abdominal pain: diagnostic accuracy study. BMJ 2009;339:b2431.

19. Rao PM, Rhea JT. Colonic diverticulitis: evaluation of the arrowhead sign and the inflamed diverticulum for CT diagnosis. Radiology 1998;209:775-9.

20. Hammond NA, Nikoladis P, Miller HF. Left Lower-Quadrant Pain: Guidelines from the American College of Radiology Appropriateness Criteria. Am Fam Physician 2010;82:766-70.

21. Rotert H, Nöldge G, Encke J, et al. The value of CT for the diagnosis of acute diverticulitis. Radiologie 2003;43:51-8.

22. Morson BC. The muscle abnormality in diverticular disease of the colon. Proc R Soc Med 1963;9:798-800.

23. Sartelli M, Catena F, Ansaloni L, et al. WSES guidelines for the management of acute left sided colonic diverticulitis in the emergency setting. World J Emerg Surg 2016;11:37.

24. Paolillo C, Spallino I. Is it safe to send home an uncomplicated diverticulitis? The DIVER trial. Intern Emerg Med 2015;10:193-4.

25. Biondo S, Golda T, Kreisler E, et al. Outpatient versus hospitalization management for uncomplicated diverticulitis. A prospective multi center randomized clinical trial (DIVER trial). Ann Surg 2014 259:1.

26. Sartelli M, Moore FA, Ansaloni L, et al. A proposal for a CT driven classification of left colon acute diverticulitis. World J Emerg Surg 2015;10:3.

27. Broderick-Villa G, Burchette RI, Collins JC, et al. Hospitalization for acute diverticulitis does not mandate routine elective colectomy. Arch Surg 2005;140:576-81.

28. Li D, Baxter NN, McLeod RS, et al. Evolving practice patterns in the management of acute colonic diverticulitis: a population-based analysis. Dis Colon Rectum 2014;57:1397-405.

29. Touzios JG. Diverticulosis and acute diverticulitis. Gastronterol Clin North Am 2009;38:513-25.

30. Galetin T, Galetin A, Vesrweber KH, Rink AD. Systematic review and comparison of national and international guidelines on diverticular disease. Int J Colorect Dis 2014;16:866-78.

31. Leifeld L, Germer CT, Bohm S, et al. S2k guidelines diverticular disease/diverticulitis. Z Gastroenterol 2014;52:663-710.
32. Stollman N, Smalley W, Hirano I. American Gastroenterological Association Institute guideline on the manage- ment of acute diverticulitis. Gastroenterology 2015;149:1944-9.

33. Binda GA, Cuomo R, Laghi A, et al. Italian Society of Colon and Rectal Surgery. Practice parameters for the treatment of colonic diverticular disease: Italian Society of Colon and Rectal Surgery (SICCR) guidelines. Techn Coloproctol 2015;19:615-26.

34. Feingold D, Steele SR, Lee S, et al. Practice parameters for the treatment of sigmoid diverticulitis. Dis Colon Rectum 2014;57:284-94.

35. Zielke A, Hasse C, Nies C, et al. Prospective evaluation of ultrasonography in acute colonic diverticulitis. Br J Surg 1997;84:385-8.

36. Bodmer NA, Thkrar KH. Evaluating the patient with left lower quadrant abdominal pain. Radiol Clin N Am 2015;53:1171-88.

37. DeStigter KK, Keating DP. Imaging update: acute colonic diverticulitis. Clin Colon Rectal Surg 2009;22:147-55.

38. Hinchey EJ, Schaal PG, Richards GK. Treatment of perforated diverticular disease of the colon. Adv Surg 1978;12:85-109.

39. Kohler L, Sauerland S, Neugebauer E. Diagnosis and treatment of diverticular disease: results of a consensus development conference. The Scientific Committee of the European Association for Endoscopic Surgery. Surg Endosc 1999;13:430-6.

40. Hall JF, Roberts PL, Ricciardi R, et al. Long-term follow-up after an initial episode of diverticulitis: what are the predictors of recurrence? Dis Colon Rectum 2011;54:238-8.

41. Chabok A, Pahiman L, Hjem F, AVOD study group. Randomized clinical trial of antibiotics in acute uncomplicated diverticulitis. Br J Surg 2012;99:532-9.

42. Nally DM, Kavanagh DO. Current controversies in the management of diverticulitis: a review. Dig Surg 2018 Apr 19 [Epub ahead of print]. doi: 10.1159/000488216.

43. Peery AF, Dellon ES, Lund J, et al. Burden of gastrointestinal disease in the United States: 2012 update. Gastroenterology 2012;143:1179-87. 